$6 \quad{ }^{a}$ Translational Social Neuroscience Unit, Department of Psychiatry, Psychosomatics, and

\section{Financial incentives facilitate the neural computation of prosocial decisions stronger in low} empathic individuals

\author{
Iotzov, Vassil ${ }^{\mathrm{a}, \mathrm{b}}$; Saulin, Anne ${ }^{\mathrm{a}}$; Kaiser, Jochen ${ }^{\mathrm{b}}$; Han, Shihui ${ }^{\mathrm{c}}$;
}

and Hein, Grit ${ }^{\mathrm{a}}$

Psychotherapy, University of Wuerzburg; ${ }^{b}$ Institute of Medical Psychology, Faculty of Medicine,

Goethe University, Frankfurt am Main; 'School of Psychological and Cognitive Sciences, PKU-

IDG/ McGovern Institute for Brain Research, Peking University, Beijing, 10008, China

\section{*corresponding authors:}

Vassil Iotzov, Translational Social Neuroscience Lab, Department of Psychiatry, Psychosomatic and Psychotherapy, University Hospital of Wuerzburg, Margarete-Höppel-Platz 1, 97080

Würzburg / Germany, Tel.: +49 93120177 412, E-mail: Iotzov_V@ukw.de

Prof. Dr. Grit Hein, Translational Social Neuroscience Lab, Department of Psychiatry, Psychosomatic and Psychotherapy, University Hospital of Wuerzburg, Margarete-Höppel-Platz 1, 97080 Würzburg / Germany, Tel.: +49 93120177 411, E-mail: $\underline{\text { Hein_G@ukw.de }}$ 


\section{Abstract}

Financial incentives are commonly used to motivate behaviours. There is also evidence

24 that incentives can decline the behaviour they are supposed to foster, for example, documented

25 by a decrease in blood donations if a financial incentive is offered. Based on these findings,

26 previous studies assumed that prosocial motivation is shaped by incentives. However, so far,

27 there is no direct evidence showing an interaction between financial incentives and a specific

28 prosocial motive. Combining drift-diffusion modelling and fMRI, we investigated the effect of

29 financial incentives on empathy, i.e., one of the key motives driving prosocial decisions. In the

30 empathy-alone condition, participants made prosocial decisions based on empathy, in the

31 empathy-bonus condition, they were offered a financial bonus for prosocial decisions, in addition

32 to empathy induction. On average, the bonus enhanced the information accumulation in empathy-

33 based decision. On the neural level, this enhancement was related to the anterior insula, the same

34 region that also correlated with empathy ratings. Moreover, the effect of the financial incentive

35 on anterior insula activation was stronger the lower a person scored on empathy. These findings

36 show that financial incentives enhance prosocial motivation in the absence of empathy but have

37 little effect on high empathic individuals.

39 Keywords

40 empathy, prosocial behaviour, incentives, drift-diffusion modelling, fMRI 


\section{Introduction}

Financial incentives are frequently used to motivate people. Such measures are based on empirical evidence showing that financial incentives increase the frequency of the rewarded behaviour (Garbers and Konradt, 2014; Wei and Yazdanifard, 2014), including cooperative and prosocial behaviours (Balliet et al., 2011; Stoop et al., 2018). For example, in a meta-analysis, Balliet and colleagues found that reward positively affects cooperation (Balliet et al., 2011).

Consequently, financial incentives could increase the motivation to behave prosocially (Ariely et $a l ., 2009)$. However, there is other evidence that incentives can undermine the very behaviour they are meant to strengthen (Titmuss, 1970; Deci et al., 1999; Benabou and Tirole, 2006;

Murayama et al., 2010; Niza et al., 2013; Rode et al., 2015; Besley and Ghatak, 2018). The most classic example in the realm of prosocial behaviours is the observation that people donate less blood if they are paid to do so, compared to the amount of blood that they donate without payment, i.e., to help others (Titmuss, 1970; Niza et al., 2013). In line with these observations, other studies have shown that adding financial incentives can reduce prosocial behaviours (Bowles, 2008; Ariely et al., 2009; Holmås et al., 2010). In sum, the evidence regarding the effects of incentives on prosocial decisions is inconsistent, and mainly based on behavioral observations that do not provide insights in the underlying motivational processes. As a result, it remained unclear whether and how financial incentives interact with a specific prosocial motive. Overcoming this limitation, our study directly investigated how a financial incentive shapes prosocial decisions that are driven by a specific prosocial motive, i.e., empathy. Incorporating previous approaches, we used a well-established decisions task (i.e., a modified version of a binary dictator game (Hein et al., 2016b)). Extending previous studies, we activated a specific prosocial motive (empathy) before participants entered the decision task, and, in one condition, added a financial incentive. This allowed us to investigate how financial incentives 
change the processing of prosocial decisions that are driven by one specific, carefully controlled 
91 empathy motive was activated again. However, before starting the decision task, the participant

92 was told that she would receive a bonus if she decided prosocially in the majority of the decision

93 trials. In the following allocation task, participants again allocated points to the respective partner

94 (here driven by empathy and the financial incentive; empathy-bonus condition). The order of the

\section{Part 1}
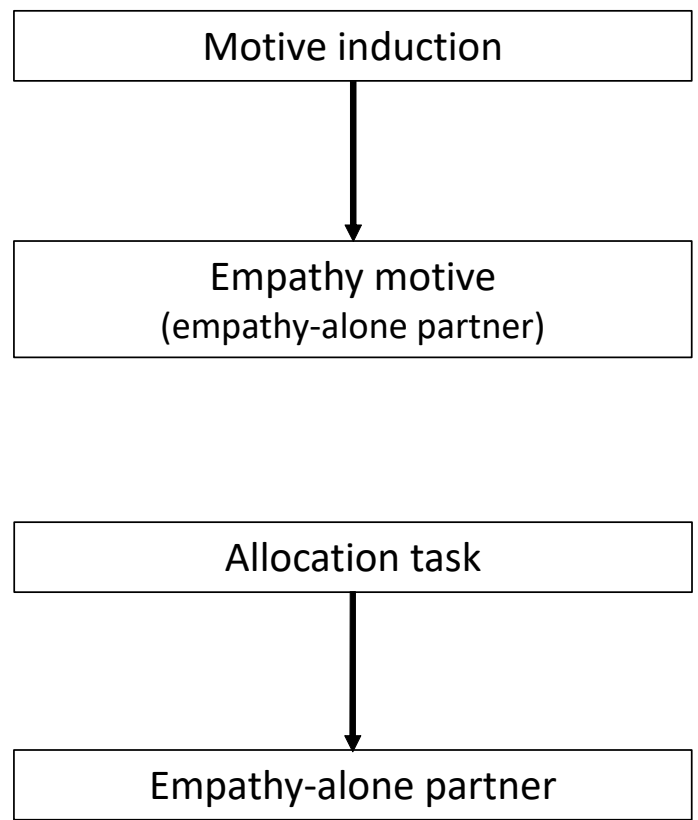

Part 2
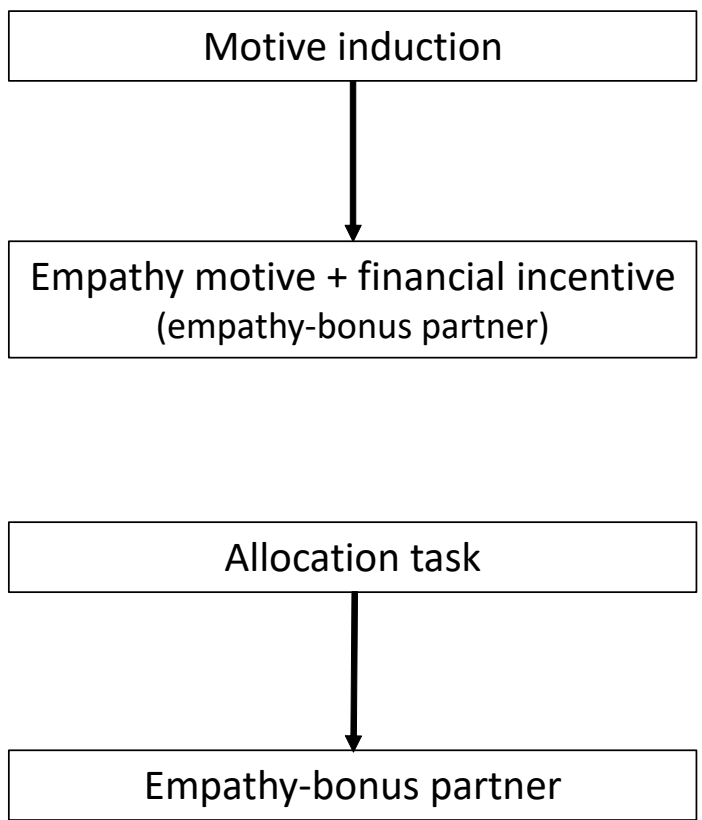

Fig. 1 Overview of an exemplary experimental procedure. The study consisted of two parts. In this example, in part 1 , the empathy motive was activated towards one confederate (the empathy-alone partner). In the following allocation task, participants allocated points to the empathy partner (i.e., driven by the empathy motive). Next, the confederate was replaced by a new individual that served as partner for part 2. Again, the empathy motive was activated. After the empathy motive induction additionally a bonus for choosing the prosocial option in the majority of trials in the subsequent allocation task was offered (empathy-bonus partner). Thus, in the following allocation task, participants allocated points towards the empathy-bonus partner (i.e., driven by the empathy motive and the additionally offered bonus). The order of motive induction (empathy-alone, empathy-bonus) was counterbalanced across participants and both confederates. The respective partner was indicated by a cue in one of two counterbalanced colors. 
To induce empathy, participants repeatedly observed two interaction partners receiving painful shocks in a number of trials, a situation known to elicit an empathic response (Lamm et

111 al., 2011; Hein et al., 2016a; Hein et al., 2016b). As a measure of the individual strength of the

112 induced empathy motive, participants rated how they felt when observing the respective other

113 person in pain (Fig. 2A). To allow participants to simulate the state (pain) of the other person, in

114 some trials, participants received painful stimulation themselves.

115 During the allocation task inside the fMRI scanner, participants allocated points to the

116 partners at a cost to themselves (Fig. 2B). The allocation of points towards the one partner

117 (empathy partner) should be based on the previously activated empathy motive (empathy-alone

118 condition). The allocation of points towards the other partner (empathy-bonus partner) was also

119 based on the previously activated empathy motive. However, in this condition, participants were

120 additionally informed that they would receive a bonus for choosing the prosocial option in the

121 majority of trials in the subsequent allocation task (empathy-bonus condition). Note that the

122 bonus corresponded to the maximally possible outcome in the allocation task (i.e., the outcome

123 that a participant would gain if she always chose the selfish option). Thus, deciding prosocially to

124 reach the bonus criterion in the empathy-bonus condition did not result in a financial loss for the

125 participants. 


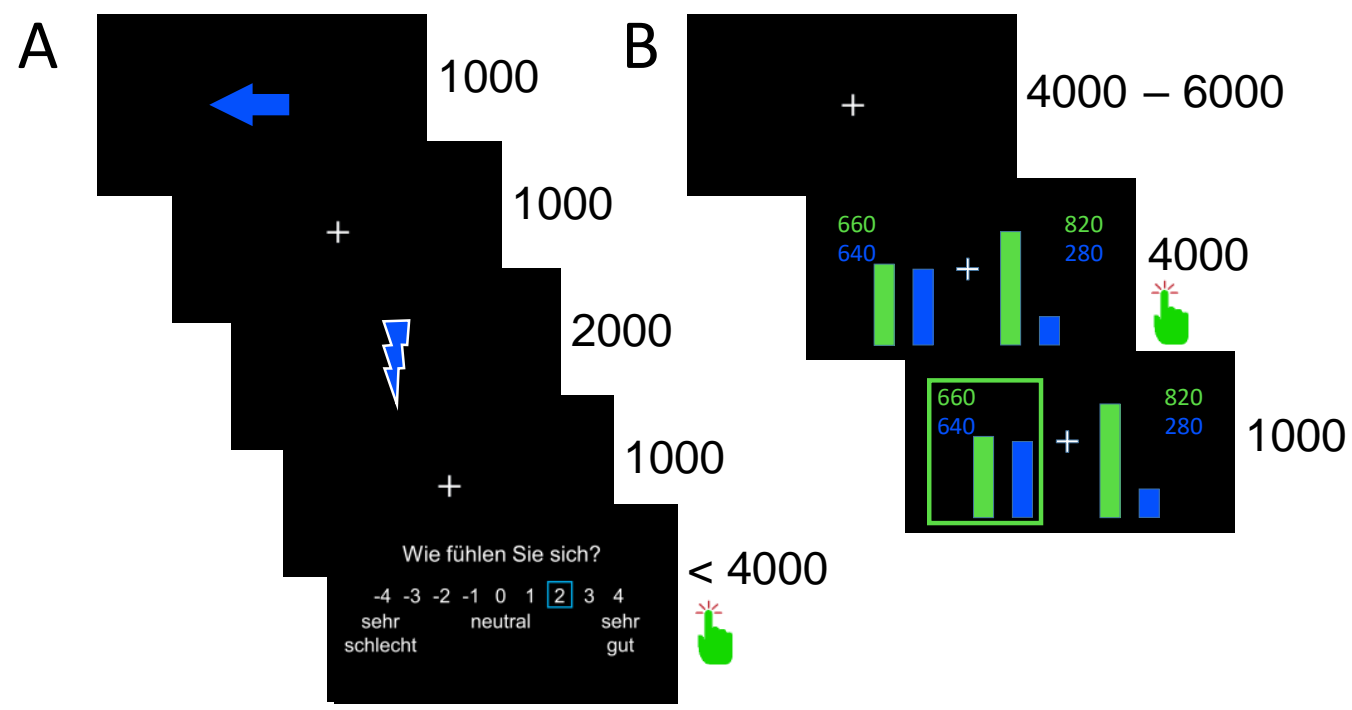

Fig. 2. Examples of induction and decision trials and schematic overview of the drift-diffusion model (DDM). A) Example trial of the empathy induction. The arrow cue indicated the receiver of the stimulation (self, the empathyalone partner in one condition or the empathy-bonus partner in the other condition). The lightning bolt indicated pain stimulation. Participants rated how they felt after observing the stimulation of the partner or receiving it themselves $(-4=$ very bad; $+4=$ very good). B) Example trial of the allocation task. Participants chose between a prosocial option that maximized points for the partner or a selfish option that maximized points for themselves. In this example trial, the participant chose the prosocial option, which maximized the outcome of the partner at a cost to the participant (green box). C) Schematic overview of the drift-diffusion model. According to the drift-diffusion model, the decision process is a noisy accumulation of information (jagged black line). From the distributions of both prosocial and selfish decisions, a set of parameters is estimated that allows to draw conclusions about the underlying cognitive processes. These are mainly the speed of information accumulation ( $v$-parameter), the starting point of the decision process ( $z$-parameter), and the amount of information to be processed ( $a$-parameter). As soon as the accumulated information reaches one of the two boundaries, the decision is made (upper boundary $=$ prosocial option; lower boundary $=$ selfish option).

To specify how incentives modulate empathy-related decisions, we used drift-diffusion modelling (DDM). DDMs assumes that during binary decisions, noisy information is 
149 the speed of noisy evidence accumulation in order to choose one of two options, i.e., the

150 efficiency of the decision process itself. Thus, a larger $v$-parameter indicates faster information

151 accumulation regarding the prosocial option. The individual decision bias is reflected by the $z-$

152 parameter. In contrast to the $v$-parameter, the $z$-parameter models the individual preferences with

153 which a person starts the decision process. For example, if a person has a strong prior preference

154 for prosocial decisions, the starting point of the decision process is closer to the prosocial

155 decision boundary, and therefore less evidence has to be accumulated regarding the prosocial

156 option. The amount of evidence that needs to be accumulated to distinguish between the two

157 options is reflected by the $a$-parameter. We modelled these three parameters $(v, z$, and $a)$ for

158 decisions that were driven by the empathy motive alone and that were driven by the combination

159 of the empathy motive and the financial incentive, based on the raw data from the entire data set

160 (i.e., including trial-by-trial information of all decisions). Additionally, the non-decision time $\left(t_{0}\right)$

161 was estimated across conditions (see Methods for details).

favour of one or another choice alternative might be shaped by affective and motivational states

164 (Roberts and Hutcherson, 2019). Supporting this assumption, affective states have been found to

165 change central parameter of the choice process such as the drift rate ( $v$-parameter; (Lerche et al.,

168 the evidence in favor of a prosocial choice might be different in different motivational states (i.e.,

169 induced by empathy and its potential interaction with the incentive), reflected by a changes in the

$170 \mathrm{drift}$ rate and/ or the starting point. 
One assumption is that financial incentives may enhance empathy-related prosocial decisions, inspired by findings of reward-related increases of prosociality (Garbers and Konradt,

173 2014; Wei and Yazdanifard, 2014). If this was true, the frequency and efficiency of prosocial

174 decisions should be higher in the empathy-bonus compared to the empathy-alone condition.

175 Specifying the potential effect of the incentive on the prosocial choice process, the DDM

176 proposes that an incentive-related facilitation of prosocial choices may originate A) from an

177 increased speed of information accumulation, i.e., an increased drift rate ( $v$-parameter (Lerche $e t$

178 al., 2018; Roberts and Hutcherson, 2019; Aylward et al., 2020; Thompson and Steinbeis, 2021),

179 B) an enhancement of participants' initial preference to choose the prosocial option, i.e, a shift of

180 the starting point towards the prosocial decision boundary (z-parameter (White et al., 2018)), or

181 C) from an enhancement of the $v$-as well as the $z$-parameter in the empathy-bonus compared to

182 the empathy-alone condition,

Alternatively, it is possible that financial incentives undermine empathy-related prosocial decisions, in line with previous findings that showed an incentive-related decrease in prosocial behaviour (Titmuss, 1970; Benabou and Tirole, 2006; Murayama et al., 2010; Rode et al., 2015).

In this case, prosocial decisions should be more frequent in the empathy-alone compared to the empathy-bonus condition. According to the DDM, such an undermining effect may be reflected

A) by a reduced speed of information accumulation ( $v$-parameter), B) a shift of the starting point away from the prosocial decision boundary ( $z$-parameter), or C) a reduction in both parameters in the empathy-bonus compared to the empathy-alone condition.

Finally, it is possible that the effect of financial incentives depends on the strength of the 
194 and changes in the drift rate and/or the starting point should be related to the individual empathy

195 ratings, i.e., the measure that captures the strength of the empathy motive during the first part of

196 the study.

197 Based on previous evidence that has linked empathy-related decisions to neural responses

198 in the AI (Hein et al., 2010; Masten et al., 2011; Hein et al., 2016b; Marsh, 2018), we assume

199 that an incentive-related increase in the $v$-and/or $z$-parameter (reflecting facilitation of empathy-

200 related decisions) is associated with an increase in brain regions associated with the processing of

201 empathy and empathy-related decisions such as the AI and the ACC. In contrast, an incentive-

202 related decrease in the $v$-and/or $z$-parameter (reflecting a potential undermining effect) should be

203 related to a decrease in AI and ACC activity.

204 Methods

205 Materials and Methods

$206 \quad$ Participant details

207

33 healthy women (mean age was 25.05 years, s.e. $=0.74$ ) participated in the study. We

208 chose a female instead of a gender-mixed subject group because it allowed us to choose female

209 confederates and thus to avoid the potential complications of gender-mixed pairing of

210 participants and confederates. The confederates were two female students, trained to play their

211 roles in counterbalanced order. The data from two participants had to be discarded as outlier

212 (frequency of prosocial decisions, 3.42 SDs below the mean $\left(M_{\text {empathy-alone }}=44.35, S D_{\text {empathy-alone }}=\right.$

213 12.97). Thus, we analyzed 31 data sets. We obtained ethics approval (EK 458122014) for

214 conducting the study and written informed consent from our participants. The experiment was

215 conducted following the Helsinki guidelines. Participants received monetary compensation (show

216 up fee plus payout from two randomly chosen trials of the allocation task; see below). 


\section{Procedure}

\section{Overall procedure}

Outside the fMRI scanner, we attached pain electrodes to the back of the participants' and the confederates' hands and determined the individual thresholds for painful and painless stimulation using a standard procedure (Hein et al., 2016a; Hein et al., 2016b). Next, the participant and the confederates played a manipulated lottery (drawing matches) that ostensibly determined the amount of pain the person would receive in the following task. Because the empathy induction required saliently more pain for the confederates, the drawing of the matches was organized in such a way that the participant always drew the last match and thus was assigned to receive only a few painful stimuli.

The participant was placed inside the fMRI scanner, and one of the confederates was placed on a chair next to the participant in the scanner room. The confederate's hand with the pain electrode was placed on a tilted table over the participants' knee. Through a mirror in the head coil, participants could see the hand of the other, together with the visual stimulation on a screen that was positioned at the end of the fMRI bed. During the empathy induction, participants either saw a dark-coloured flash (painful stimulation) or a light-coloured flash (non-painful stimulation), indicating the intensity of the stimulation of the confederate. In a small portion of trials (five from fifteen), they received pain stimulation themselves, indicated by a dark-coloured flash of a different colour. During the decision task, participants were presented two options to allocate points between themselves and the other person. Colours were counterbalanced across participants.

The study started with the empathy induction, followed by the allocation task towards the first confederate. After replacing this confederate, the same procedure (empathy induction followed by the allocation task) was repeated with the second confederate (Fig. 1). In the 
241 empathy-alone condition, the allocation task started immediately after the empathy induction. In

242 the empathy-bonus condition, after the empathy induction, participants were told that they would

243 receive a bonus (additional 5 Euro) if they chose the prosocial option in the majority of trials. We

244 deliberately refrained from specifying the percentage of prosocial decisions that were required to

245 win the bonus to avoid strategy effects. However, participants knew that the bonus would

246 compensate the maximally possible outcome in the allocation task (i.e., the outcome that a

247 participant would gain if she always chose the selfish option). Thus, deciding prosocially to reach

248 the bonus in the empathy-bonus condition did not result in a financial loss for the participants. To

249 minimize reputation effects, participants received the bonus information in private without the

250 partner's knowledge.

Apart from the bonus in the empathy-bonus condition, the experimental procedure was

252 identical in both conditions. The order of the conditions and the assignment of the confederates

was counterbalanced across participants. At the end of the experiment, both confederates left, and

the participants stayed in the scanner until anatomical image acquisition was completed. Finally,

participants were asked to complete the Interpersonal Reactivity Index (IRI; (Davis, 1980)), and a

scale that assessed their impression of both confederates (Hein et al., 2016a). The impression

ratings were comparable between confederates $\left(\operatorname{lmm} \chi^{2}(1)=0.36, p=.55, B=-0.10\right.$, s.e. $\left.=0.16\right)$.

Participants spent approximately $60 \mathrm{~min}$ inside the scanner, and the entire procedure

260 randomly chosen allocation trials, and the bonus of five Euro if they had made prosocial

261 decisions in $75 \%$ of all trials.

All ratings during the induction phase and all decisions in the allocation task were kept 
264 the following: Inside the scanner room, the partner had a separate visual display, such that the

265 participant viewed stimuli via back-projection from a mirror onto a screen, while the confederates

266 beside the scanner viewed stimuli via cardboards/video glasses with a built-in display (Hein $e t$

$267 a l ., 2016 a)$. Thus, all ratings and decisions were private and could not be observed by the other

268 participants (Hein et al., 2016a). Moreover, participants knew that they would not meet after the

269 experiment because the scanned participant needed to stay longer for an anatomical scan. The

270 experimenter was outside the scanner room, and it was pointed out that he could not see the

271 ratings and decisions either.

In each empathy-induction trial, first we presented a coloured arrow indicating the person who will receive the following electric stimulation for $1000 \mathrm{~ms}$. After this cue, a fixation cross was presented for $1000 \mathrm{~ms}$, followed by a coloured lightning bolt shown for $2000 \mathrm{~ms}$.

276 Participants were informed that a blinking dark-coloured lightning bolt indicates a painful

277 stimulus, whereas a blinking light-coloured lightning bolt indicates a non-painful stimulus. After

278 receiving or observing the electric stimulation, we showed a 9-point rating scale with the question

279 "How do you feel?". The scale ranged from -4 (labeled "very bad") to +4 (labeled "very good").

Participants had to respond within 4000 ms (Fig. 2A). The empathy induction consisted of 30

trials: 10 that were ostensibly painful for the partner (other-pain trials), 5 that were not painful for painful trials (self-no-pain trials) for the participant. The self-pain trials were added to allow participants to simulate the state (pain) of the other person. To test their potential influence on 
pain trials that were preceded by an other-pain trial (i.e., empathy ratings without preceding self-

pain experience). The results showed no difference between the other-pain ratings after self-pain and the other-pain ratings without prior self-pain $\left(\mathrm{t}_{(61)}=0.34, \mathrm{p}=.73\right)$. Based on these results, the self-pain experience had no significant effect on empathy changes during empathy induction.

\section{Allocation task}

respective partner (Fig. 2B) and could choose between maximizing the relative outcome of the

relative outcome at a cost to the partner (selfish choice). The outcome was relative to the outcome

points was always higher for the participant compared to the partners. This measure was inspired

by previous behavioral economics research, showing that participants make more prosocial prosocial choices, which was the main focus of our study. 
points, which corresponds exactly to the gain of the partner (400 - $100=300$ points). We used

these fixed and symmetrical ratios to minimize unspecific effects of loss aversion.

participant and the potential gain for the current partner. Participants had to choose one of two

\section{Pain stimulator}

For pain stimulation, we used electrical stimulation (bipolar, monophasic; output range

$3275 \mathrm{~Hz}, 0-10 \mathrm{~mA}$ ) from a single-current stimulator (Neurometer CPT/C; Neurotron Inc.). After

328 attaching the electrodes at the index finger of the right hand and connecting them to the single-

329 current stimulator, the respective person was asked to press the button for defining the current

330 threshold and to decide when she is feeling the stimulation - the value of this threshold was used

331 as painless stimulation. In a second run the participant was asked to press the same button, but 
now to hold it pressed until the pain was at an unacceptable level and then to release - this

threshold was used for the painful stimulation.

\section{Experimental design and statistical analyses}

The aim of our study was to compare prosocial decisions driven by empathy alone with

used a within-subject design in which each participant performed the identical social decision

task under two different conditions: the empathy-bonus and the empathy-alone condition.

Behavioural data were analyzed with R-Studio Version 1.1.463(RStudio Team, 2020) and R

Version 3.6.0(RCore Team, 2019) and Python (HDDM; Spyder Version 3.3.2; Python Version

2.7.15 (Van Rossum, 2007; Wiecki et al., 2013)).

All regression analyses were performed with the R-packages "stats" (RCore Team, 2019)

using, "Ime4" (Bates et al., 2015), "car" (Fox and Weisberg, 2019), and MuMIn (Bartoń, 2019).

Results were visualized with the "tidyverse" package (Wickham et al., 2019). All continuous

predictors in our regressions are z-scored.

the data was log-transformed to normal distribution. Pearson correlation was computed between

349 the empathy ratings and the empathic concern scale (EC) from the Interpersonal Reactivity Index

350 (IRI)(Davis, 1980). In further data analyses, we used linear models within condition and linear 
We choose the DDM, because of its small but trackable number of key parameters and

358 in the existing literature. We used hierarchical drift-diffusion modelling (HDDM

359 (Vandekerckhove et al., 2011; Wiecki et al., 2013)), which is a version of the classical driftdiffusion model that exploits between-subject and within-subject variability using Bayesian parameter estimation methods and thus is ideal for use with relatively small sample sizes. The analyses were conducted using the python implementation of HDDM (Wiecki et al., 2013).

Based on previous studies showing changes in drift rate (Lerche et al., 2018; Roberts and (White et al., 2018) if decisions are made in different affective states, we assumed that these two parameters might also be affected by motivational states. However, given that the modulation of affect and motivation is not the same, effects on the third parameter (the $a$-parameter) are also possible. Therefore, we estimated the full model with $v, z$, and $a$ possibly being modulated by our two conditions. Moreover, we estimated the non-decision parameter $\left(t_{0}\right)$, which indicates the duration of all extradecisional processes like basic encoding or motor processes (Voss et al., 2004). In paradigms like ours that used an identical experimental setting across conditions, it was recommended to estimate the $t_{0}$-parameter across conditions (Wagenmakers et al., 2008; Servant

373 et al., 2014; Nunez et al., 2017). Following this recommendation, we estimated the $t_{0}$-parameter

374 across the empathy-bonus and the empathy-alone conditions (mean $t_{0}=0.58$, s.e. $=0.02$ ), and

375 refrained from estimating it for each condition separately (see full HDDM results table at 376 github.com (https://github.com/Vassil-Iotzov/empathy_incentives)). 
We conducted the same DDM analyses with two different inputs. In one analysis, the

378 input of the DDM was defined categorically based on the type of response $(1=$ prosocial option;

$3790=$ selfish option). In the other analyses, we used the trial-by-trial point difference (self-loss or

380 other-gain) as additional covariate effecting the drift rate to estimate a hierarchical random

381 intercept model (see Chen and Krajbich (2018) for a similar approach). Other input parameters

382 were reaction time (in seconds), condition (empathy-bonus, empathy-alone), and participants

383 number (0 to 30$)$.

To evaluate the model fit, we conducted posterior predictive checks by comparing the observed data with 500 datasets simulated by our model, thus using the method that has been particularly recommended for HDDMs to obtain quantile comparison and 95\% credibility

(Wiecki et al., 2013)). The respective quantile comparison table is provided at github.com

checked by visual inspection of the estimation chain of the posteriors, as well as computing the alone condition. 
(1) $P(-\mid a, z, v)=\frac{e^{-(2 v a)}-e^{-(2 v z)}}{e^{-(2 v a)}-1}$

402 (Alexandrowicz, 2018) in the basic formula:

(2) $P(-\mid a, z, v)=\frac{e^{-\left(\frac{2 v a}{s^{2}}\right)}-e^{-\left(\frac{2 v z}{s^{2}}\right)}}{e^{-\left(\frac{2 v a}{s^{2}}\right)}-1}$ Iotzov/ggddm).

410 scanner (Siemens Healthineers), equipped with a one-channel Siemens head coil. Scanner noise 411 was reduced with soft foam earplugs, and head motion was minimized with foam pads. Stimuli

412 presented in the induction phase and in the allocation task were projected onto a rear projection

413 screen located in the front of the scanner. Behavioural responses were recorded with a five-key

414 fibre-optic response box placed on the right hand, and when necessary, vision was corrected

415 using MRI-compatible lenses that matched the dioptre of the participant. Structural image

416 acquisition consisted of 176 T1-weighted transversal images (voxel size of $1 \mathrm{~mm}$ ) (Hein et al.,

417 2016a). Functional imaging data was collected during the allocation task, using T2*-weighted

418 echo-planar imaging (32 slices, slice thickness of $3 \mathrm{~mm}$, ascending acquisition; repetition time,

$4192100 \mathrm{~ms}$; echo time, $30 \mathrm{~ms}$; flip angle, $80^{\circ}$; field of view, $240 \mathrm{~mm}$; matrix, $80 \times 80$ ). In every 


\section{Preprocessing and statistical model}

The images were analyzed with SPM12 (Functional Imaging Laboratory, 2019) and

Matlab version 8.6 (Matlab, 2015). Images were preprocessed following the standard procedure

recommended in the SPM manual (Functional Imaging Laboratory, 2019), including realignment,

slice time correction, coregistration, segmentation, normalize, smoothing.

First-level analyses were performed with the general linear model (GLM), using a

canonical hemodynamic response function (HRF). For each of the conditions (empathy-alone and empathy-bonus condition), the respective regressors of prosocial choice trials were included as regressors of interest. The prosocial decisions regressor spanned the period from the onset of the decision screen until the participants' reaction (average of $1146.37 \mathrm{~ms}$ ). Regressors of no interest included the period from the participants' reaction to decision offset (average of $2853.63 \mathrm{~ms}$ ) and the immediately following period showing the participants' decision (1000 ms).

Sixteen of our participants made less than five selfish decisions in at least one condition.

To avoid empty cells in the model, we refrained from computing direct contrasts between prosocial and selfish choices, and selfish choices were included as regressor of no interest.

For the second-level analyses, contrast images for comparisons of interest (empathybonus > implicit baseline, empathy-alone > implicit baseline, empathy-bonus > empathy-alone, and empathy-alone > empathy-bonus) were initially computed on a single-subject level. In the next step, the individual images of the main contrast of interest (empathy-bonus > implicit baseline) were regressed against the $v$-parameter. Results were thresholded using 5\% family wise error (FWE) corrected voxel-based inference. We also conducted exploratory analyses using 5\% FWE cluster-based inference with a cluster-forming threshold of $\mathrm{P}_{\text {uncorrected }}<.001$ and a minimal cluster size of $\mathrm{k}=50$ and used this threshold for the visualization of our results. Beta estimates 
444 were extracted from the entire clusters of activation in the anterior insula obtained from 5\% FWE

445 cluster-based inference with $\mathrm{P}<.001$ cluster-forming threshold, $\mathrm{k}=50$, using MarsBaR (Brett,

446 2002). Moreover, the respective beta-estimates were extracted from an independent region of

447 interest, defined based on a $20 \mathrm{~mm}$ sphere around the peak coordinates $(\mathrm{x}=-43 ; \mathrm{y}=14 ; \mathrm{z}=7)$

448 from a significant activation likelihood cluster found across all pain empathy experiments in a

449 current meta-analysis (Jauniaux et al., 2019).

\section{Code and data availability}

Behavioural data and scripts are available at github.com (https://github.com/Vassil-

452 Iotzov/empathy_incentives). Imaging data are available at neurovault.org

453 (https://identifiers.org/neurovault.collection:7568).

\section{$454 \quad$ Results}

455 Empathy was induced with comparable strength in both conditions.

To quantify the strength of the induced empathy, we calculated the participants' trial-by-

457 trial ratings while observing the partner in pain relative to their self-pain ratings. Comparing the

458 ratings between the empathy-alone and the empathy-bonus condition revealed no significant

459 differences between conditions $\left(\operatorname{lmm} \chi_{(1)}^{2}=0.0001, p<.99, B=-0.002\right.$, s.e. $\left.=0.22, R_{m}^{2}<.01\right)$,

460 indicating that empathy was induced with comparable strength in the empathy-alone and the

461 empathy-bonus condition.

The average of the individual empathy ratings in both conditions, i.e., our measure of state 
According to these results, the induced motive is related to empathic concern rather than personal

468 distress.

469 The financial incentive increased the frequency of prosocial decisions, in particular if empathy

470 was low.

Comparing the reaction times of prosocial decisions in the empathy-bonus and the

472 empathy-alone condition revealed no significant difference, $\left(\operatorname{lmm} \chi_{(1)}^{2}=2.24, p=.13, B=0.27\right.$,

473 s.e. $=0.18)$. There was also no difference when only selfish decisions were considered $\left(\operatorname{lmm} \chi^{2}(1)\right.$

$474=0.14, p=.71, B=-0.08$, s.e. $=0.22)$ and when all decisions were included $\left(\operatorname{lmm} \chi^{2}(1)=1.99, p\right.$

$475=.16, B=0.26$, s.e. $=0.19)$.

The frequency of prosocial decisions was significantly higher in the empathy-bonus

477 condition compared to the empathy-alone condition (Fig. 3A), $\left(\operatorname{lmm} \chi_{(1)}^{2}=14.35, p<.01, B=-\right.$

4780.57 , s.e. $\left.=0.15, R^{2}{ }_{m}=.08\right)$. We also computed the percent change in prosocial decisions in the

479 empathy-bonus condition relative to the empathy-alone condition ((empathy-bonus - empathy-

480 alone)/empathy-alone *100). The results revealed a significant relative increase of $23.88 \%($ s.e. $=$ $7.91 \%), t_{(30)}=3.02, p<.01$.

In an additional analysis, we compared the number of prosocial decisions in the empathy-

483 alone condition with the number of prosocial decisions in a baseline condition (without any motive induction) from a previous study using a similar paradigm and the same allocation task

485 (Hein et al., 2016b). The results revealed significantly more prosocial decisions in the empathy-

486 alone condition compared to the baseline condition, empathy-alone $(M=73.92 \%$, s.e. $=0.39)$,

487 baseline condition $(M=49.37 \%$, s.e. $=0.32),\left(t_{(59.963)}=4.85, \mathrm{p}<.01\right)$.

We tested if that was induced before the decision task. A linear mixed model with 
490 (empathy-alone / empathy-bonus) and empathy ratings $\times$ condition as predictors revealed a

491 significant negative effect of empathy ratings $\left(\operatorname{lmm} \chi_{(1)}^{2}=6.61, p=.01, B=-0.36\right.$, s.e. $\left.=0.17\right)$,

492 which was comparable in both conditions, condition $\left(\operatorname{lmm} \chi_{(1)}^{2}=2.17, p=.14, B=0.27\right.$, s.e. $=$

$4930.18)$, condition $\mathrm{x}$ empathy rating interaction $\left(\operatorname{lmm} \chi_{(1)}^{2}=0.02, p=.89, B=-0.02\right.$, s.e. $=0.18 ; R^{2}{ }_{m}$

$494=.15)$. According to these results, higher empathy ratings predicted faster prosocial decisions.

495 A regression analysis with the percentage change in prosocial decisions as dependent

496 variable and empathy ratings as predictor revealed a significant negative relationship $(B=-0.42$,

497 s.e. $\left.=0.17, p=.02, R^{2}=.18\right)$. The lower an individual's empathy ratings, the stronger the increase

498 in the frequency of prosocial decisions in the empathy-bonus condition relative to the empathy-

499 alone condition (Fig. 3B).

500

501

502

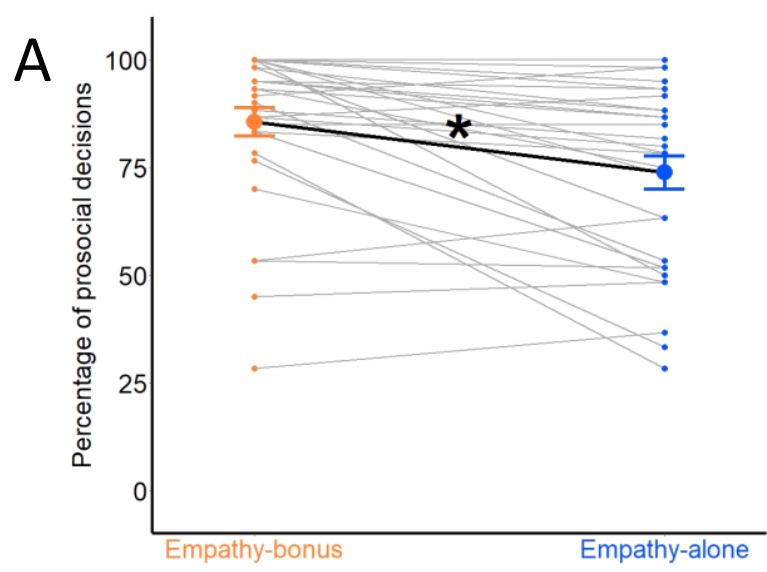

503

504

505

506

507

508

509

510

511

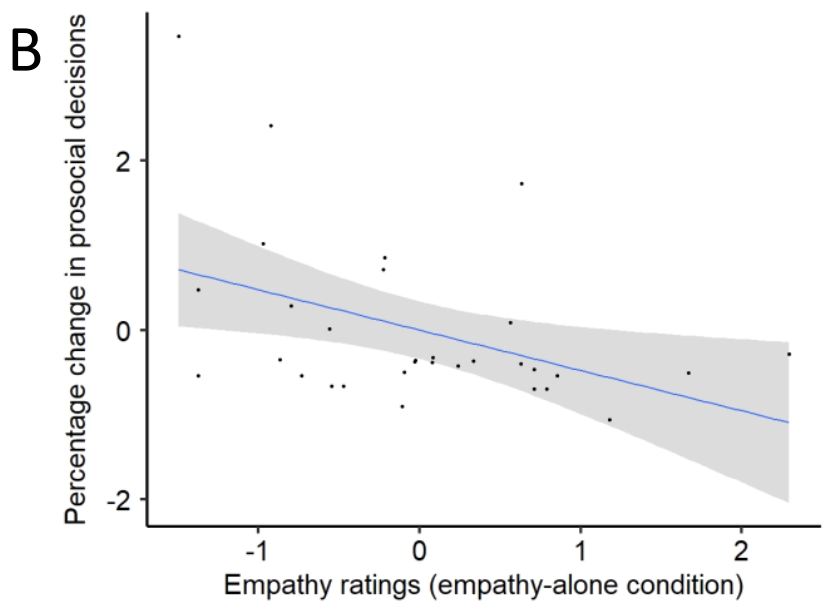

Fig. 3. Percentage of prosocial decisions, reaction times and the relationship between the relative increase in prosocial decisions in the empathy-bonus condition and empathy ratings. A) Individual percentage of prosocial decisions in the empathy-bonus (orange) and the empathy-alone condition (blue). B) Negative relationship between the relative increase in prosocial decisions in the empathy-bonus condition and empathy ratings. The lower a participant's empathy rating, the higher the incentive-related increase in prosocial decisions. 
512 The financial incentive increased the speed of information accumulation, but not the initial

513 decision preference.

514 To specify which component of the prosocial decision process was enhanced by the

515 financial incentive, relative to prosocial decisions in the empathy-alone condition, we used

516 hierarchical drift-diffusion modelling (HDDM; (Vandekerckhove et al., 2011; Wiecki et al.,

517 2013)), a version of the classical drift-diffusion model that exploits between-subject and within-

518 subject variability using Bayesian parameter estimation methods. We estimated the three

519 aforementioned DDM parameters $(v, z, a)$ for every condition and participant. Comparing the

520 observed data with 500 datasets simulated by the HDDM (Wiecki et al., 2013) showed that the

521 HDDM fit the data with $95 \%$ credibility (see quantile comparison table at github.com

522 (https://github.com/Vassil-Iotzov/empathy_incentives).

We compared the speed of information accumulation (drift rate; $v$-parameters), the initial

524 prosocial decision preferences (starting point; $z$-parameters), and the amount of integrated

525 information ( $a$-parameters) between the empathy-bonus and the empathy-alone condition. The

526 comparison of the posteriors (Wiecki et al., 2013) revealed high probability for a larger $v$ -

527 parameter in the empathy-bonus condition compared to the empathy-alone condition, $v$-empathy-

528 bonus $(M=2.03$, s.e. $=0.22)$, v-empathy-alone $(M=1.24$, s.e. $=0.19),\left(p_{(v \text {-empathy-bonus }>v \text {-empathy- }}\right.$

529 alone $)=.99$; Fig. 4A). In contrast, the probability for a differences between the other decision

530 parameters was relatively low, $z$-empathy-bonus $(M=0.47$, s.e. $=0.01), z$-empathy-alone $(M=$

0.46 , s.e. $\left.=0.01 ; p_{(z \text {-empathy-bonus }>\text {-empathy-alone })}=.54\right), a$-empathy-bonus $(M=1.96$, s.e. $=0.08), a$ -

532 empathy-alone $\left(M=1.88\right.$, s.e. $\left.=0.09 ; p_{(\text {a-empathy-bonus }>\text { a-empathy-alone })}=.79\right)$. This indicates that

533 financial incentives enhanced the efficiency of the prosocial decision process, while leaving

534 initial prosocial preferences unchanged. 
537 vs other into account. To do so, we added the point difference (point for self vs points for other)

538 as additional covariate effecting the drift rate (Chen and Krajbich, 2018). The results replicated

539 the observed findings (high probability for a larger $v$-parameter in the empathy-bonus condition

540 compared to the empathy-alone condition: $v$-empathy-bonus $(M=5.69$, s.e. $=0.22), v$-empathy-

541 alone $(M=4.94$, s.e. $\left.=0.19), p_{(v \text {-empathy-bonus }>\text { v-empathy-alone })}=.99\right)$, no differences between the other

542 decision parameters $z$-parameter: $z$-empathy-bonus $(M=0.49$, s.e. $=0.01), z$-empathy-alone $(M=$

5430.47 , s.e. $=0.01), p_{(z \text {-empathy-bonus }>z \text {-empathy-alone })}=.70 ; a$-parameter: $a$-empathy-bonus $(M=1.97$,

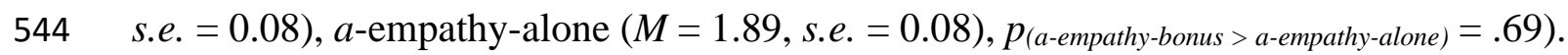

546 The incentive-related facilitation of prosocial decisions and individual differences in empathy are

547 associated with changes in anterior insula activation.

First, we conducted the main contrasts between the prosocial decision-related activation

549 in the empathy-bonus vs the empathy-alone conditions and vice versa. Based on the applied

550 statistical threshold $\left(\mathrm{P}_{(\mathrm{FWEvoxel}-\mathrm{based})}<.05\right)$ there were no significant results. This indicates that on

551 average the same neural circuitries are involved in computing prosocial decisions driven by

552 empathy and by empathy and the bonus.

Second, we identified neural regions that are related to an increase in drift rate in the

554 empathy-bonus condition, i.e., the choice parameter that accounted for the facilitation of the

555 prosocial decision process in the empathy-bonus compared to the empathy-alone condition. We

556 regressed the individual $v$-parameters against the neural activation during prosocial decisions in

557 the empathy-bonus condition, using a second-level regression. The results showed significant 

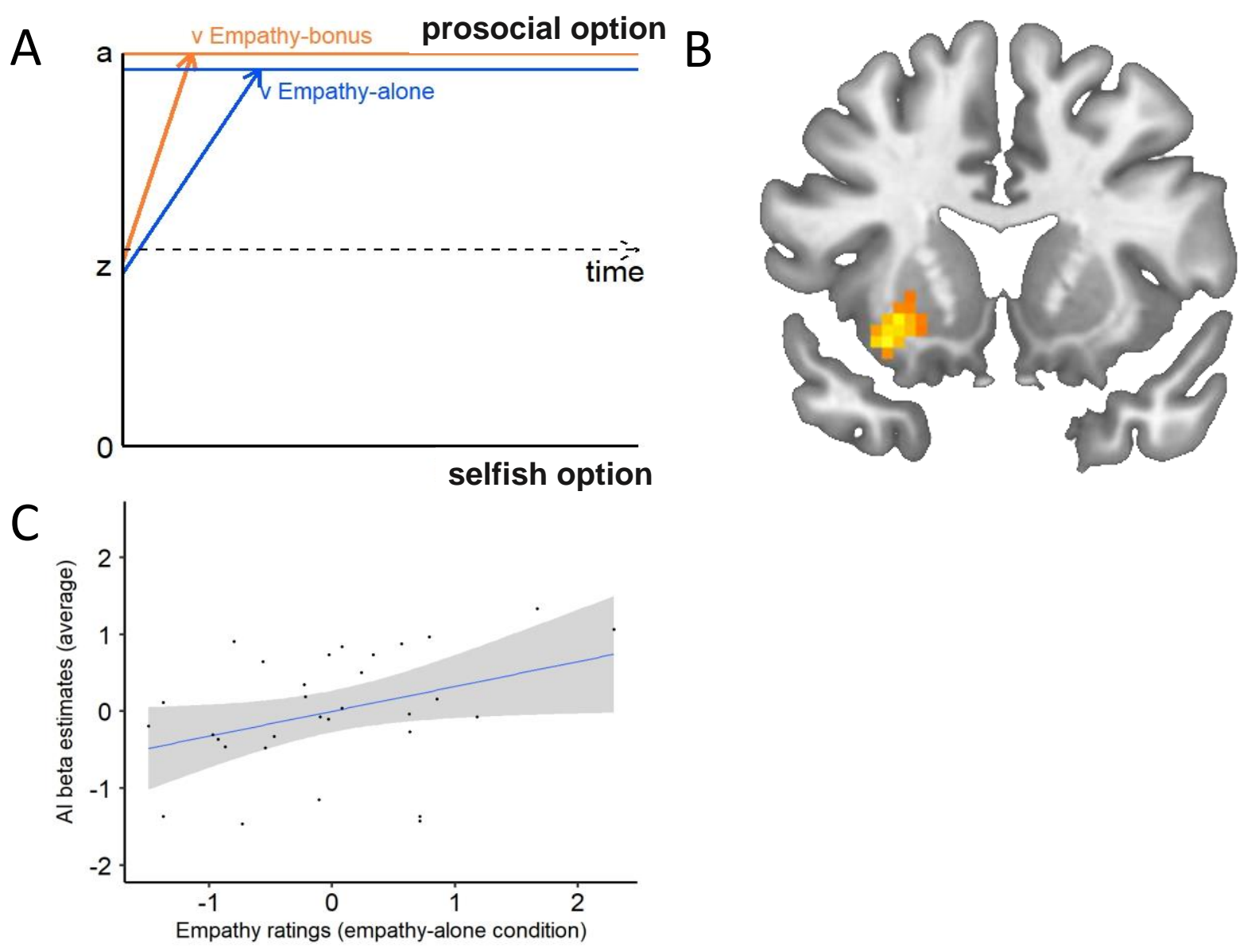

Empathy ratings (empathy-alone condition)

Fig. 4. Drift-diffusion modelling (DDM) results and their relationship with neural responses in anterior insular cortex and empathy ratings. A) Visualization of the obtained DDM parameters showing an enhanced speed of information accumulation ( $v$-parameter) in the empathy-bonus condition (orange) compared to the empathy-alone condition (blue). B) The neural response in the anterior insula (AI) correlates with the individual $v$-parameters in the empathybonus condition (visualized using $5 \%$ FWE cluster-based inference with $\mathrm{P}<.001$ cluster-forming threshold; $\mathrm{k}=50$ ). The higher the speed of information accumulation in the empathy-bonus condition, the stronger the neural response in AI. C) Significant positive relationship between the individual strength of the AI response and the individual empathy ratings. The beta estimates reflect the average of AI activation from the empathy-bonus and the empathyalone condition, extracted from the same AI clusters that correlated with the $v$-parameter in the empathy-bonus condition (shown in B). 


\begin{tabular}{|c|c|c|c|c|c|}
\hline Region & Hemisphere & $\mathbf{x} \mathbf{y} \mathbf{z}$ & Cluster size & $t$-value & $\mathbf{P}\left(\mathbf{F W E} \mathbf{E}_{\text {cluster-based }}\right)$ \\
\hline \multirow[t]{2}{*}{ Anterior Insula } & Left & -27385 & 97 & 6.24 & $.007 *$ \\
\hline & Left & $-3014-13$ & 59 & 4.86 & .048 \\
\hline Lingual gyrus & Right & $24-67-1$ & 384 & 5.95 & $.000 *$ \\
\hline Inferior lingual & Left & $-51-58-19$ & 181 & 5.23 & .000 \\
\hline \multicolumn{6}{|l|}{ gyrus } \\
\hline Pallidum & Left & $-18-7-1$ & 66 & 4.64 & .032 \\
\hline
\end{tabular}

584 individual differences in empathy (Hein et al., 2010; Lamm et al., 2011; Marsh, 2018), we tested

585 if the observed AI region (i.e., the region that correlated with the speed of information

586 accumulation in the empathy-bonus condition) was also related to the empathy ratings that we

587 collected prior to the allocation task. To do so, we extracted the average of the beta estimates

588 related to prosocial decisions in the empathy-bonus and the empathy-alone condition from the

589 entire activated AI clusters and regressed them against the individual differences in empathy

590 ratings. The results showed a significant positive effect of empathy ratings $(B=0.41$, s.e. $=0.19$,

$\left.591 p=.04, R^{2}=.14\right)$. Because we used the average of the beta estimates from AI across both 
conditions, we can infer that the observed AI activation, in general processes individual

593 differences in empathy, i.e., unbiased by the specific experimental conditions. The higher a

594 participant's empathy ratings, the stronger the neural response in the AI region, i.e. the same

595 region that correlated with the speed of information accumulation in the empathy-bonus

condition (Fig. 4C).

597 The financial incentive has a differential effect on anterior insular activation in high and low

598 empathic individuals.

599 Given that the $v$-parameter and empathy ratings both are processed in the same AI region,

600 it is plausible to assume that the two variables interact. To test that we conducted a linear mixed

601 model with the beta estimates of AI activation during prosocial decisions in the empathy-bonus

602 and the empathy-alone condition as a dependent variable. The individual $v$-parameters and

603 empathy ratings were added as predictors, condition (empathy-bonus / empathy-alone) was added

604 as a categorical variable. The results revealed significant main effects of condition $\left(\operatorname{lmm} \chi_{(1)}^{2}=\right.$

$12.26, p<.01, B=0.67$, s.e. $=0.19)$, empathy ratings $\left(\operatorname{lmm} \chi^{2}(1)=4.43, p=.04, B=0.33\right.$, s.e. $=$

$0.16)$ and the $v$-parameter $\left(\operatorname{lmm} \chi_{(1)}^{2}=25.60, p<.01, B=0.68\right.$, s.e. $\left.=0.13\right)$. Moreover, there

were significant interactions between empathy ratings $\mathrm{x} v$-parameter $\left(\operatorname{lmm} \chi^{2}(1)=5.60, p=.02, B\right.$

$608=-0.40$, s.e. $=0.17)$, and condition $\mathrm{x} v$-parameter $\left(\operatorname{lmm} \chi_{(1)}^{2}=4.23, p=.04, B=-0.41\right.$, s.e. $=$

$0.20)$, but not between condition $\mathrm{x}$ empathy ratings $\left(\operatorname{lmm} \chi_{(1)}^{2}=0.14, p=.71, B=0.08\right.$, s.e. $=$ interaction $\left(\operatorname{lmm} \chi^{2}(1)=10.75, p<.01, B=0.70\right.$, s.e. $\left.=0.21, R_{m}^{2}=.49\right)$. 
with the beta estimates extracted from the pallidum, right lingual gyrus and left inferior lingual $=0.21)$, right lingual gyrus $\left(\operatorname{lmm} \chi_{(1)}^{2}=0.91, p=.34, B=-0.20\right.$, s.e. $\left.=0.21\right)$, left inferior lingual significant main effects of $v(B=0.70$, s.e. $=0.11, p<.01)$ and empathy ratings $(B=0.29$, s.e. $=$ the $v$-parameter $\left(B=0.25\right.$, s.e. $=0.18, p=.19 ; R^{2}=.31$, Fig. 5B $)$. ratings and AI beta estimates separately in the empathy-bonus and the empathy-alone condition.

637 Given that empathy facilitates prosocial decisions (Batson et al., 1995; Decety et al., 2016) and 
639 empathy ratings and the drift ratings and empathy ratings and AI activation. To test these apriori

640 assumptions, we used one-sided tests (Pfaffenberger and Patterson, 1977; Ruxton and Neuhäuser,

641 2010). In the empathy-alone condition, the results revealed a significant positive relationship

642 between $v$-parameter and AI beta estimates $(B=0.38$, s.e. $=0.19, p=.02$, Fig. 5F $)$, a significant

643 positive relationship between empathy ratings and AI beta estimates $(B=0.43$, s.e. $=0.18, p=$

644.01 , Fig. 5D), and a significant positive relationship between empathy ratings and drift rate $(B=$

0.30$, s.e. $=0.18, p=.05)$. In the empathy-bonus condition we observed a significant positive

relationship between $v$-parameter and AI beta estimates $(B=0.73$, s.e. $=0.12, p<.01$, Fig. $\mathbf{5 E})$,

647 while the relationships between empathy ratings and AI beta estimates $(B=0.23$, s.e. $=0.16, p=$

648.08 , Fig. 5C) and between empathy ratings and drift rate were not significant $(B=0.21$, s.e. $=$

$6490.17, p=.10)$. The finding of a positive relationship between empathy ratings and the drift rate

650 and empathy ratings and AI beta estimates in the empathy-alone condition is in line with previous

651 evidence showing that empathy facilitates prosocial decisions (Batson et al., 1995; Decety et al.,

652 2016). In the empathy-bonus condition, the relationship between empathy ratings and drift rate

653 and empathy ratings and AI estimates was no longer significant, indicating that in the presence of

654 an incentive, empathy was no longer a significant driver of prosocial decisions. Interestingly, the

655 interaction between the empathy ratings and the drift rate reduced AI activation in the empathy-

656 bonus condition while increasing it in the empathy-alone condition. This indicates that in the

657 empathy-bonus condition the empathy ratings (indicating the strength of the empathy motive

658 before the bonus was offered) suppress the positive effect of the $v$-parameter on the neural

659 response in AI.

660 

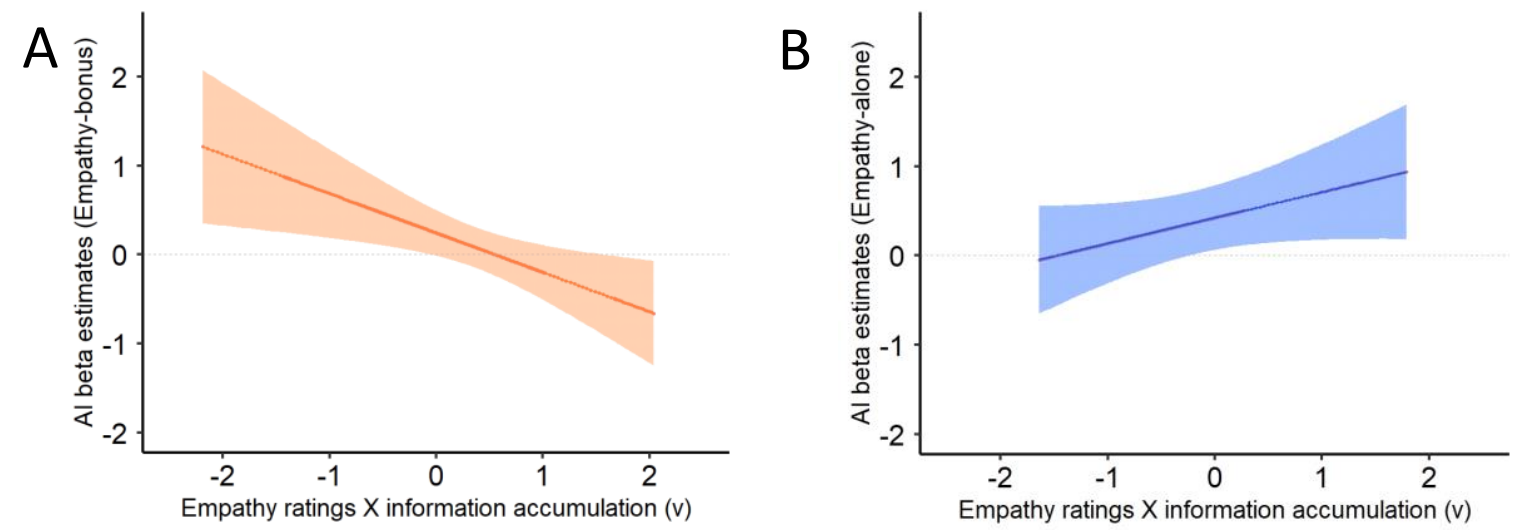

664
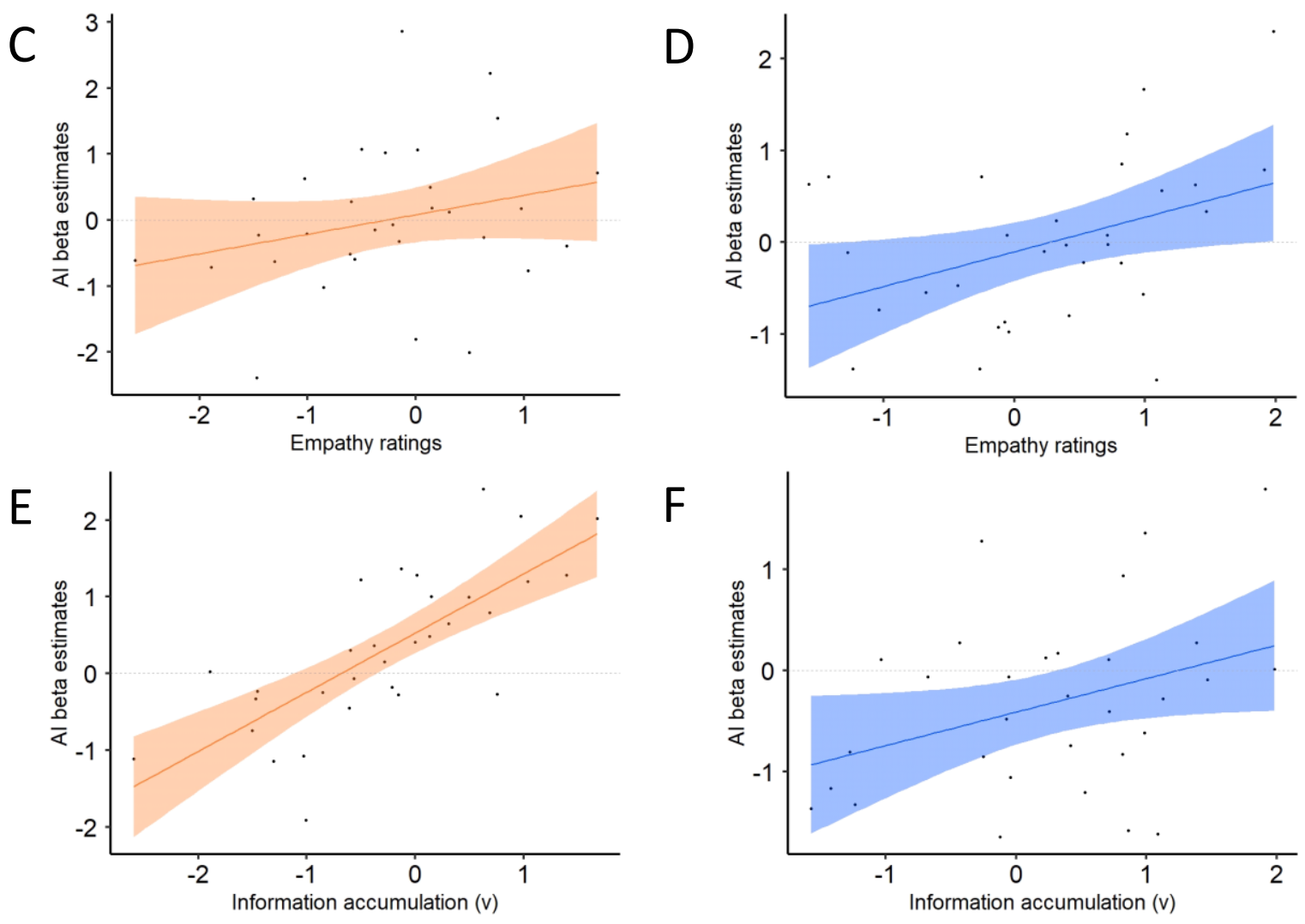

Fig. 5. Relationships between anterior insula (AI) beta estimates and empathy ratings and between AI beta estimates and speed of information processing ( $v$-parameter) in the empathy-bonus and empathy-alone conditions. The beta estimates reflect the average of AI activation from the empathy-bonus and the empathy-alone condition, extracted from the same AI clusters that correlated with the $v$-parameter in the empathy-bonus condition (shown in Fig. 4B). A) Effect of the empathy ratings $\mathrm{x} v$-parameters interaction on AI responses in the empathy-bonus condition. B) Effect of the empathy ratings $\mathrm{x} v$-parameters

672 interaction on AI responses in the empathy-alone condition. C) The relationship between the individual 673 strength of the AI responses and the individual empathy ratings in the empathy-bonus condition was not 674 significant. D) Significant positive relationship between the individual strength of the AI responses and 675 the individual empathy ratings in the empathy-alone condition. E) Significant positive relationship 
676 between the individual strength of the AI responses and the speed of information processing ( $v$-parameter)

677 in the empathy-bonus condition. F) Significant positive relationship between the individual strength of the

678 AI responses and the speed of information processing ( $v$-parameter) in the empathy-alone condition.

679

680

681

682

To test the robustness of the differential effects in the empathy-bonus and the empathyalone conditions, we extracted the beta-estimates of prosocial decision-related activation in the empathy-bonus and the empathy-alone condition from an independent region of interest in the AI (defined based on the peak coordinates reported in a recent meta-analysis on empathy of pain studies (Jauniaux et al., 2019). We conducted a linear mixed model with these beta-estimates as dependent variable, and condition (empathy-bonus / empathy - alone), empathy ratings, and $v$ parameters as predictors. The results replicated the significant condition $\mathrm{x} v$-parameter $\mathrm{x}$ empathy rating interaction reported above $\left(\operatorname{lmm} \chi^{2}(1)=5.81, p=.02, B=0.61\right.$, s.e. $\left.=0.25, R_{m}^{2}=.19\right)$, reflecting a negative relationship in the empathy-bonus condition and a positive relationship in the empathy-alone condition.

\section{Discussion}

Our study investigated how financial incentives affect empathy-related prosocial decisions. The results show that on average financial incentives increase the frequency of prosocial decisions (Fig. 3A), in particular in individuals that scored low on empathy (Fig. 3B).

The finding that the financial bonus enhanced the frequency of prosocial decisions is in line with previous studies showing an incentive-related increase in prosocial behaviours (Balliet et al., 2011; Stoop et al., 2018). Extending this previous evidence, our results reveal that this effect is modulated by individual differences in empathy, i.e., stronger if a person's empathic motivation is low. Besides providing insights into the interplay between financial incentives and empathy, our results specified how financial incentives affect the prosocial decision process. The results of 
drift-diffusion modelling showed that the financial incentive enhanced the efficiency (i.e., speed

703 significant effect on participants' initial prosocial preferences, i.e., the preference of making a

704 selfish or prosocial decision with which they entered the decision process (captured by the $z$ -

705 parameter).

706 Outside the domain of prosocial decisions, there is evidence that the efficiency of

et al., 2018; Roberts and Hutcherson, 2019; Aylward et al., 2020; Thompson and Steinbeis, greater state anxiety show increased $v$-parameter on fearful face trials. Extending these findings, our results reveal that the speed of information accumulation is shaped by the motivation that drive participants' prosocial decisions, i.e., higher if a prosocial decision is rewarded than if it is only based on empathy.

720 (captured by an increased speed of information accumulation) is related to an increase of AI 
Adding a novel aspect, our findings reveal how financial incentives alter the effect of

724 empathy on the computation of prosocial decisions in the anterior insular cortex. After offering a

725 bonus in the empathy-bonus condition, the relationship between empathy ratings and drift rate

726 and empathy ratings and AI estimates was no longer significant, indicating that in the presence of

727 an incentive, empathy was no longer a significant driver of prosocial decisions. Interestingly, the

728 interaction between the empathy ratings and the drift rate significantly reduced AI activation in

729 the empathy-bonus condition (Fig. 5A) while increasing it in the empathy-alone condition (Fig.

730 5B). This indicates that in the empathy-bonus condition, the strength of the empathy motive

731 (captured by the individual strength of the empathy ratings before the bonus was offered)

732 suppressed the positive relationship between information accumulation during prosocial decisions

733 and the neural response in AI. Together, these findings indicate that the anterior insula integrates

734 self-regarding (gaining the financial incentive) and other-regarding (empathy with the other

735 person) motives that both elicit prosocial decisions and thus forms a plausible neural basis for the

736 impact of financial incentives on empathic motivation.

In our study, empathy was conceptualized as a motive that can drive prosocial decisions.

738 And indeed, the empathy ratings of our participants that correlated with empathic concern (but

739 not personal distress) facilitated the prosocial decision process in the empathy-alone condition, in

740 line with previous findings (Batson et al., 1995; Decety et al., 2016). That said, the result that

741 financial incentives counteracted the facilitating effect of empathy on prosocial decisions in

742 highly empathic individuals might indicate that highly empathic individuals are less motivated to

743 empathize in the presence of an incentive, an assumption that supports the notion that empathy

744 itself is a motivated state (Zaki, 2014).

745 The financial incentive for prosocial decisions was offered in private, and self-image

746 concerns were reduced as far as possible, at least with regard to public reputation. However, 
747

748

749

750

751

752

753

754

755

756

757

758

759

760

761

762

763

764

765

766

767

768

769

770

771

772

773 some highly empathic participants nevertheless showed an incentive-related decline in prosocial decisions (see also Fig. 3B). It is conceivable that highly empathic participants feel insulted by the bonus because "being paid to be nice" undermined their intrinsic empathic motivation that otherwise (i.e., in the empathy-alone condition) drives their prosocial decisions. Thus, although on average our findings show that the incentive increased the frequency of prosocial decisions compared to an empathy-alone condition, it is still possible that it undermines prosocial behavior in highly empathic participants. To test this assumption, future studies should test the effect of financial incentives on empathy-based decisions in extreme groups, i.e., groups of extremely high or low empathic individuals. Moreover, it would be interesting to use a trial-by-trial bonus manipulation that allows for modelling the effect directly as part of the DDM.

In summary, our current results indicate that financial incentives offered in private facilitate prosocial decisions in low empathic individuals but have little effect in case of strong empathic motivation.

\section{References}

Alexandrowicz, R. W. (2018) 'The diffusion model visualizer: an interactive tool to understand the diffusion model parameters', Psychological Research.

Ariely, D., Bracha, A. \& Meier, S. (2009) 'Doing good or doing well? Image motivation and monetary incentives in behaving prosocially', American Economic Review, 99(1), pp. 544-555.

Aylward, J., Hales, C., Robinson, E. \& Robinson, O. J. (2020) 'Translating a rodent measure of negative bias into humans: the impact of induced anxiety and unmedicated mood and anxiety disorders', Psychological medicine, 50(2), pp. 237-246.

Balliet, D., Mulder, L. B. \& Van Lange, P. A. M. (2011) 'Reward, punishment, and cooperation: A meta-analysis', Psychological Bulletin, 137(4), pp. 594-615.

Bartoń, K. (2019) 'MuMIn: Multi-Model Inference, R package version 1.43.15'. 

emotion a source of altruistic motivation?', Journal of personality and social psychology, 40(2),

778 pp. 290-302. psychology, 68(2), p. 300.

Benabou, R. \& Tirole, J. (2006) 'Incentives and prosocial behavior', American Economic Review, 96(5), pp. 1652-1678. decision making: a formal analysis of models of performance in two-alternative forced-choice tasks', Psychological review, 113(4), pp. 700-765.

Bolton, G. E. \& Ockenfels, A. (2000) 'ERC: A Theory of Equity, Reciprocity, and Competition', 1609.

Brett, M. A., Jean-Luc; Valabregue Romain; Poline, Jean-Baptiste (2002) 'Region of interest analysis using an SPM toolbox', 8th International Conference on Functional Mapping of the Human Brain, Sendai, Japan, NeuroImage.

Chen, F. \& Krajbich, I. (2018) 'Biased sequential sampling underlies the effects of time pressure and delay in social decision making', Nature Communications, 9(1), p. 3557.

801 Davis, M. H. (1980) 'A multidimensional approach to individual differences in empathy', JSAS

802 Catalogue of Selected Documents in Psychology, 10.

803 Decety, J., Bartal, I. B.-A., Uzefovsky, F. \& Knafo-Noam, A. (2016) 'Empathy as a driver of

804 prosocial behaviour: highly conserved neurobehavioural mechanisms across species', 
805

806

807

808

809

810

811

812

813

814

815

816

817

818

819

820

821

822

823

824

825

826

827

828

829

830

831

832

833

834

835

Philosophical transactions of the Royal Society of London. Series B, Biological sciences, 371(1686), pp. 20150077-20150077.

Deci, E. L., Koestner, R. \& Ryan, R. M. (1999) 'A meta-analytic review of experiments examining the effects of extrinsic rewards on intrinsic motivation', Psychol Bull, 125(6), pp. 627668; discussion 692-700.

Eklund, A., Nichols, T. E. \& Knutsson, H. (2016) 'Cluster failure: Why fMRI inferences for spatial extent have inflated false-positive rates', Proceedings of the National Academy of Sciences, 113(28), pp. 7900-7905.

Exley, C. (2017) 'Incentives for prosocial behavior: The role of reputations', Management Science, 64(5), pp. 2460-2471.

Fehr, E. \& Schmidt, K. M. (1999) 'A Theory of Fairness, Competition, and Cooperation*', The Quarterly Journal of Economics, 114(3), pp. 817-868.

Forstmann, B. U., Ratcliff, R. \& Wagenmakers, E. J. (2016) 'Sequential Sampling Models in Cognitive Neuroscience: Advantages, Applications, and Extensions', Annual review of psychology, 67, pp. 641-666.

Fox, J. \& Weisberg, S. (2019) An R Companion to Applied Regression, Thousand Oaks CA, Sage.

Functional Imaging Laboratory, W. T. C. f. N., Institute of Neurology, UCL (2019) 'SPM12 Manual'.

Garbers, Y. \& Konradt, U. (2014) 'The effect of financial incentives on performance: A quantitative review of individual and team-based financial incentives', Journal of Occupational and Organizational Psychology, 87(1), pp. 102-137.

Gelman, A. \& Rubin, D. B. (1992) 'Inference from Iterative Simulation Using Multiple Sequences', Statistical Science, 7(4), pp. 457-472.

Hein, G., Engelmann, J. B., Vollberg, M. C. \& Tobler, P. N. (2016a) 'How learning shapes the empathic brain', Proceedings of the National Academy of Sciences, 113(1), pp. 80-85.

Hein, G., Morishima, Y., Leiberg, S., Sul, S. \& Fehr, E. (2016b) 'The brain's functional network architecture reveals human motives', Science, 80(351), pp. 1074-1078.

Hein, G., Silani, G., Preuschoff, K., Batson, C. D. \& Singer, T. (2010) 'Neural responses to ingroup and outgroup members' suffering predict individual differences in costly helping', Neuron, 68(1), pp. 149-160. 
839 Hutcherson, C. A., Bushong, B. \& Rangel, A. (2015) 'A Neurocomputational Model of Altruistic 840 Choice and Its Implications', Neuron, 87(2), pp. 451-462.

841 Jauniaux, J., Khatibi, A., Rainville, P. \& Jackson, P. L. (2019) 'A meta-analysis of neuroimaging 842 studies on pain empathy: investigating the role of visual information and observers' perspective', 843 Social Cognitive and Affective Neuroscience, 14(8), pp. 789-813.

844 Lamm, C., Decety, J. \& Singer, T. (2011) 'Meta-analytic evidence for common and distinct 845 neural networks associated with directly experienced pain and empathy for pain', NeuroImage, $846 \quad$ 54(3), pp. 2492-2502.

847 Lerche, V., Neubauer, A. B. \& Voss, A. (2018) 'Effects of implicit fear of failure on cognitive 848 processing: A diffusion model analysis', Motivation and Emotion, 42(3), pp. 386-402.

Marsh, A. A. (2018) 'The neuroscience of empathy', Current opinion in behavioral sciences, 19, 850 pp. 110-115.

851 Masten, C. L., Morelli, S. A. \& Eisenberger, N. I. (2011) 'An fMRI investigation of empathy for 852 'social pain' and subsequent prosocial behavior', NeuroImage, 55(1), pp. 381-388.

Matlab (2015) version 8.6 (R2015b), Natick, Massachusetts, The MathWorks Inc.

857 Niza, C., Tung, B. \& Marteau, T. M. (2013) 'Incentivizing blood donation: Systematic review and 858 meta-analysis to test Titmuss' hypotheses', Health Psychology, 32(9), pp. 941-949.

859 Nunez, M. D., Vandekerckhove, J. \& Srinivasan, R. (2017) 'How attention influences perceptual 860 decision making: Single-trial EEG correlates of drift-diffusion model parameters', Journal of 861 mathematical psychology, 76(Pt B), pp. 117-130.

862 Pfaffenberger, R. C. \& Patterson, J. H. (1977) Statistical methods for business and economics, 863 RD Irwin.

864 Ratcliff, R., Smith, P. L., Brown, S. D. \& McKoon, G. (2016) 'Diffusion Decision Model:

865 Current Issues and History', Trends in cognitive sciences, 20(4), pp. 260-281. 
RCore Team (2019) 'R: A Language and Environment for Statistical Computing', Vienna, Austria.

Roberts, I. D. \& Hutcherson, C. A. (2019) 'Affect and Decision Making: Insights and Predictions from Computational Models', Trends in cognitive sciences, 23(7), pp. 602-614. incentives in conservation policy: A review of the empirical evidence', Ecological Economics, 117, pp. 270-282.

RStudio Team (2020) 'RStudio: Integrated Development Environment for R', Boston, MA.

Ruxton, G. D. \& Neuhäuser, M. (2010) 'When should we use one-tailed hypothesis testing?', 875 Methods in Ecology and Evolution, 1(2), pp. 114-117. Insight in model constraints based on psychological laws', Cognitive Psychology, 72, pp. 162195. games', Journal of Environmental Economics and Management, 88(C), pp. 300-310.

Thompson, A. \& Steinbeis, N. (2021) 'Computational modelling of attentional bias towards threat in paediatric anxiety', Developmental Science, n/a(n/a), p. e13055.

Van Rossum, G. (2007) 'Python programming language', USENIX annual technical conference.

Vandekerckhove, J., Tuerlinckx, F. \& Lee, M. D. (2011) 'Hierarchical diffusion models for twochoice response times', Psychological methods, 16(1), pp. 44-62. of Criterion Shifts in the Lexical Decision Task', Journal of memory and language, 58(1), pp. 140-159. 
White, C. N., Liebman, E. \& Stone, P. (2018) 'Decision mechanisms underlying mood-congruent

898 Wickham, H., Averick, M., Bryan, J., Chang, W., McGowan, L., François, R., Grolemund, G., 899 Hayes, A., Henry, L., Hester, J., Kuhn, M., Pedersen, T., Miller, E., Bache, S., Müller, K., Ooms, 900 J., Robinson, D., Seidel, D., Spinu, V. \& Yutani, H. (2019) 'Welcome to the Tidyverse', Journal 901 of Open Source Software, 4, p. 1686.

Wiecki, T. V., Sofer, I. \& Frank, M. J. (2013) 'HDDM: Hierarchical Bayesian estimation of the drift-diffusion model in Python', Frontiers in neuroinformatics, 7, p. 14.

904 Woo, C. W., Krishnan, A. \& Wager, T. D. (2014) 'Cluster-extent based thresholding in fMRI 905 analyses: pitfalls and recommendations', NeuroImage, 91, pp. 412-419.

Zaki, J. (2014) 'Empathy: a motivated account', Psychological Bulletin, 140(6), p. 1608.

908 Funding

909 This work was supported by the German Research Foundation (HE 4566/2-1; HE 4566/5-1).

\section{Acknowledgments}

911 We thank Isabelle Ehrlich for help with data collection and Marthe Gründahl and Martin Weiß

912 for feedback on the manuscript.

\section{Authors' Contributions}

914 Grit Hein and Vassil Iotzov designed the research with input from Jochen Kaiser; Vassil Iotzov

915 programmed the experiment with input from Anne Saulin and performed the research; Vassil

916 Iotzov and Anne Saulin analyzed the data with input from Grit Hein, Jochen Kaiser and Shihui

917 Han; Grit Hein and Vassil Iotzov wrote the paper with input from Anne Saulin, Jochen Kaiser

918 and Shihui Han.

\section{Author Affiliations}

920 Vassil Iotzov, Translational Social Neuroscience Lab, Department of Psychiatry, Psychosomatic 921 and Psychotherapy, University Hospital of Wuerzburg, Margarete-Höppel-Platz 1, 97080

922 Würzburg / Germany, Tel.: +49 93120177 412, E-mail: Iotzov_V@ukw.de 
923 Anne Saulin, Translational Social Neuroscience Lab, Department of Psychiatry, Psychosomatic

924 and Psychotherapy, University Hospital of Wuerzburg, Margarete-Höppel-Platz 1, 97080

925 Würzburg / Germany, Tel.: +49 93120177 412, E-mail: Saulin_A@ukw.de

926 Prof. Dr. Jochen Kaiser, Institute of Medical Psychology, Goethe-Universität, Heinrich-

927 Hoffmann-Str. 10, Building 93A, Room A45, 60528 Frankfurt am Main, Tel.: 069-6301 6302, E-

928 mail: J.Kaiser@med.uni-frankfurt.de

929 Prof. Dr. Shihui Han, Culture and Social Cognitive Neuroscience Lab, School of Psychological 930 and Cognitive Sciences, PKU-IDG/McGovern Institute for Brain Research, Peking University,

931 Room 1614, Wang Kezhen Building, No. 52, Haidian Road, Haidian District, Beijing 100080,

932 People's Republic of China, Tel: (86) 10-6275-9138, E-mail: shan@pku.edu.cn

933 Prof. Dr. Grit Hein, Translational Social Neuroscience Lab, Department of Psychiatry,

934 Psychosomatic and Psychotherapy, University Hospital of Wuerzburg, Margarete-Höppel-Platz

935 1, 97080 Würzburg / Germany, Tel.: +49 93120177 411, E-mail: Hein_G@ukw.de

\section{Competing interests}

937 The authors declare no competing interests. 